\title{
Caminos hacia la corresponsabilidad: los varones en el cuidado infantil en Uruguay*
}

Sol Scavino Solari** Karina Batthyány***

\begin{abstract}
Resumen
El presente artículo busca conocer cuáles son las representaciones de cuidado de varones que actualmente tienen hijos menores de 6 años en Uruguay. Partiendo de la idea de que existe un desajuste entre los discursos (más equitativos) y las prácticas (aún inequitativas) sobre la participación de los varones en el cuidado, se analizaron los discursos sobre el cuidado que los varones desarrollan, desde una perspectiva de género y corresponsabilidad. Se identifican tres tipos de paternidades actuales: tradicionales, de involucramiento leve y con tendencia a la corresponsabilidad.
\end{abstract}

Palabras clave: $\quad$ Paternidades, Cuidados, Género, Corresponsabilidad.

* Recibido el 21 de junio de 2018, aceptado el 30 noviembre de 2018.

** Investigadora Asistente del Grupo de Investigación de Sociología de Género del Departamento de Sociología, Facultad de Ciencias Sociales, Universidad de la República, Montevideo, Uruguay. solscavino@gmail.com / http://orcid.org/0000-0002-6675-7765?lang=en

*** Coordinadora del Grupo de Sociología de Género del Departamento de Sociología de la Facultad de Ciencias Sociales (UDELAR), Montevideo, Uruguay. karina.batthyany@cienciassociales.edu.uy / http://orcid.org/0000-0001-68369806 
Paths to Co-Responsibility: Men in Child Care in Uruguay

\begin{abstract}
This article seeks to know which are the representations of care of men who currently have children under 6 years in Uruguay. Starting from the idea that there is a mismatch between the (more equitable) discourses and the (still inequitable) practices on male participation in care, the discourses on care that men develop, from a gender perspective, were analyzed and co-responsibility. Three types of current paternities are identified: traditional, of slight involvement and with a tendency to coresponsibility.
\end{abstract}

Keywords: Paternity, Care, Gender, Co-Responsibility. 
Los varones, las masculinidades y el cuidado

Al menos en los últimos 50 años en América Latina, la demanda por la transformación sociocultural de las desigualdades de género ha sido sin duda una de las más relevantes, expresándose en la expresión de los movimientos sociales y políticos que han colocado demandas específicas en la agenda pública.

Las mismas se vinculan a la promoción de un cambio en las identidades masculinas y femeninas, así como de los dispositivos culturales e institucionales que contribuyen a su reproducción.

Si bien a nivel cultural los cuestionamientos a los modelos hegemónicos de masculinidad y de feminidad están presentes en la arena pública, esto no es tan claro en cuanto a la redistribución del trabajo remunerado y no remunerado, las responsabilidades y el poder.

En América Latina, Uruguay es el primer país en contar con un Sistema Nacional Integrado de Cuidados que tiene como objetivo la reorganización social del cuidado, así como la modificación de la actual división sexual del trabajo.

En este contexto emerge la pregunta acerca de cómo los varones están viviendo sus paternidades vinculadas al cuidado no remunerado de los niños/as en este país, teniendo en cuenta que los antecedentes cuantitativos y cualitativos de investigación señalan una disonancia entre discursos y prácticas en torno a la corresponsabilidad (Barker; Aguayo, 2011:33; Batthyány; Ferrari; Scavino, 2015:161). A pesar de esta distancia, estudios comparativos en Brasil, Chile y México muestran que los varones que son discursivamente más equitativos en género, también lo son en sus prácticas de cuidados, aunque la mayoría de los padres (varones) se declara "ayudantes" de las mujeres, alcanzando al 80\% en México (Barker; Aguayo, 2011:35). Los antecedentes también muestran que los padres varones cuyos padres estuvieron involucrados en su crianza tienden a involucrarse más en el cuidado de sus hijos (Barker; Aguayo, 2011:34). 
En Uruguay, si bien el tiempo de cuidados y de trabajo doméstico sigue siendo repartido de manera fuertemente desigual entre varones y mujeres, la instalación de demandas y conflictos planteados en las parejas es un hecho, particularmente en las de dobles ingreso y en donde ambos miembros de la pareja tienen inserciones laborales similares y niveles educativos altos (Batthyány; Perrotta; Scavino, 2017:78).

La posibilidad de cambios, acuerdos y conflictos en las negociaciones sobre la participación de varones y mujeres en el cuidado, muestran que tanto la maternidad como la paternidad son una "construcción sociocultural en rápido proceso de transformación, aunque sometidas a una dinámica en la que coexisten el avance y la resistencia al cambio" (Tobío et alii, 2010:104).

En este marco, el presente artículo se propone comprender cómo significan las paternidades, los varones que son actualmente padres de niños/as de 6 años. Se realiza un análisis de contenido de los discursos de varones de distintos niveles socioeconómicos de una muestra intencional realizada en Montevideo, luego de caracterizar a los padres actuales para enmarcar las producciones discursivas en algunos datos generales en base a la Encuesta de Uso del Tiempo (2013).

\section{Representaciones, prácticas y políticas públicas vinculadas a la paternidad}

En Uruguay, país en el que se desarrolla el estudio, los avances del Sistema Nacional Integrado de Cuidados en generar mecanismos para la redistribución se expresan en al menos dos grandes ejes: el aumento de los centros de cuidado infantil (que no garantizan la corresponsabilidad entre varones y mujeres, pero liberan tiempo de cuidado) y la modificación en los subsidios de cuidado. La Ley 19.161 amplía de 12 a 14 semanas la licencia por maternidad (obligatoria), extiende la licencia paternal (que puede llegar a 13 días en el caso de trabajadores dependientes de la actividad privada) y establece para los/as trabajadores/as de la 
actividad privada y algunos de la actividad pública, un subsidio de medio horario para el cuidado, que puede ser ejercido tanto por el padre como por la madre hasta los seis meses del hijo o la hija. Estas últimas dos licencias no tienen carácter obligatorio. La licencia parental es transferible entre la madre y el padre, pudiendo fraccionar su duración entre ambos en forma alternada (Batthyány; Genta; Perrotta, 2018:13).

Respecto a los centros de cuidado infantil, en el país los niños/as de 4 a 6 años tienen una cobertura universal y de 0 a 3 años ha aumentado la cobertura total de $49.1 \%$ en 2014 a $52.1 \%$ en 2016, incrementando la oferta pública y disminuyendo levemente la privada (SNIC, 2017:29).

La división sexual del trabajo es una práctica social que se recrea constantemente basándose en los roles y estereotipos de género presentes en las culturas, sociedades e instituciones. Los contextos institucionales influyen en las parejas y sus planes de cuidado en al menos tres aspectos: ofreciendo incentivos y desincentivos, creando más o menos oportunidades y estableciendo referencias de tipo cultural (Risman, 1999, en Abril et alii, 2015:6).

Un claro ejemplo acerca de cómo la división sexual del trabajo está instituida es la diferencia ente la licencia por paternidad y maternidad y las dificultades de que los varones tomen el medio horario, cuando esto es posible en las leyes (Romero-Balsas, 2015:89). Por ejemplo, en Uruguay solo 2,6\% de los varones hace uso del medio horario (Batthyány; Genta; Perrotta, 2015:31). Este aspecto se vincula con el hecho de que los mercados laborales también responden a lógicas masculinas que tienden a reproducir los roles actuales de género, generando obstáculos para la participación de las mujeres en los mismos, así como para la dedicación de los varones al cuidado de los hijos/as recién nacidos.

En la literatura se reconoce la existencia de un cambio que ha llevado a hablar de que, en las transiciones a la paternidad, se encuentran señales de transformación de las pautas tradicionales (Barbeta-Viñas; Cano, 2017:15). Esta búsqueda de nuevas 
masculinidades y paternidades ha sido acompañada con el desarrollo de conceptos como el de "paternidad sostenible" que implica un

\begin{abstract}
compromiso a través del tiempo, más allá de la infancia de los hijos y con independencia de las relaciones de pareja o las circunstancias vitales o sociales en que el padre y la madre se vean inmersos. (Alberdi; Escario, 2007, en Tobío et alii, 2010:105).
\end{abstract}

Sin embargo, aún se identifican barreras culturales para la participación de los varones en el cuidado: por los modelos de masculinidad hegemónica (Connell, 1995, en Comas, 2016:11) y por la desigual participación de varones y mujeres en el mercado laboral, lo que no contribuye a generar incentivos para que los primeros se involucren más en el cuidado (Himmelweit; Land, 2011, en Comas, 2016:12).

Por otro lado, varios estudios han mostrado que existe una distancia entre las representaciones de género (más equitativas) y las prácticas en el uso del tiempo (Barker; Aguayo, 2011:33; Hakim, 2005:80; CIS, 2004b:1, en Tobío, 2010:105; Batthyány; Ferrari; Scavino 2015:161-162).

En el caso uruguayo, las representaciones sociales de varones y mujeres jóvenes (de 14 a 29 años) son más equitativas que sus prácticas expresadas en la distribución del tiempo que dedican al cuidado y al trabajo doméstico no remunerado.

Por ejemplo, un cuarto de los varones jóvenes está de acuerdo con que el cuidado de los hijos sea una tarea primordial de las mujeres, lo que indica que casi un $75 \%$ de los varones jóvenes considera que también se trata de una tarea primordial de los varones. Aproximadamente el $90 \%$ de los varones jóvenes considera que "las mujeres deberían compartir las tareas del hogar con los varones para desarrollarse tanto en la esfera doméstica como "fuera del hogar'". Los grados de acuerdo son diferentes si se considera el nivel educativo de los jóvenes y sus posicionamientos socioeconómicos, siendo que a menor nivel 
socioeconómico las representaciones obedecen más al modelo clásico de mujer ama de casa y varón proveedor. Por ejemplo, un tercio de los jóvenes más pobres está de acuerdo con que las tareas de la casa han de ser asumidas sobre todo por las mujeres (Batthyány; Ferrari; Scavino, 2015:154).

Contrariamente, las Encuestas de Uso del Tiempo muestran que la corresponsabilidad presente en las representaciones sobre el trabajo no remunerado se desdibuja, ya que las mujeres jóvenes participan del TNR en un $18 \%$ más y dedican el doble de tiempo (16 horas más) que los varones para la realización del mismo (Batthyány; Ferrari; Scavino, 2015:151). También se observa que las brechas en la participación y el tiempo dedicado al trabajo no remunerado son más altas en los jóvenes de hogares con menores ingresos, en relación a los de mayores ingresos.

A pesar de que la situación en general es la adopción de un discurso equitativo, de género, políticamente correcto que no se refleja en el tiempo y la participación en el trabajo no remunerado por parte de los varones, existe un pequeño grupo de padres que efectivamente han cambiado su comportamiento.

Según Abril et alii (2015:15) los "nuevos padres" se encuentran con limitaciones institucionales como son la escasez de centros de cuidados infantiles públicos, aunque estos padres son conscientes de que van "contra corriente" en lo que refiere a las diversas instituciones como el mercado de trabajo, sistema educativo, sanitario, entre otras, dado que aún no es frecuente el modelo de padres cuidadores. Tobío (2010:108) afirma que los padres implicados en el cuidado de manera igualitaria representan un futuro minoritario, pero que van en una línea coherente con los cambios familiares descritos al principio por los que han transitado las sociedades occidentales.

Otra de las limitantes es la postura de las propias mujeres quienes aseguran que son ellas las que mantendrán la responsabilidad última y el control sobre los cuidados, monopolizando las tareas de cuidado y limitando el margen de actuación paterna (Allen; Hawkins, 1999; Faunt, 2008, en Abril et alii, 2015:15; Scavino, 2017:155). 
En los antecedentes, se señala que, para aumentar la participación de los padres en el cuidado, son necesarias políticas de cuidados (licencias por paternidad más extendidas y accesibles para los varones, individuales e intransferibles (Batthyány; Genta; Perrotta, 2018). También políticas socioeducativas, que la paternidad sea promovida desde sectores como la salud y la educación. Se señala la incorporación de talleres y programas experienciales como Paternidad Activa en (CIDE, Chile), Programa $\mathrm{H}$ con hombres jóvenes (Brasil, México, Chile, entre otros) o el Programa P (Brasil, Chile, Nicaragua). Esto debería ser acompañado por una política integral que busque transformar las relaciones sociales de género: aumentando la participación de las mujeres en el mercado laboral, disminuyendo la brecha salarial, aumentando la oferta pública de centros de cuidado infantil, políticas de trabajo decente, políticas de salud de los varones, entre otras (Aguayo; Barker; Ekimelman, 2016:101).

En este contexto, y visto que Uruguay es el primer país en Latinoamérica en desarrollar un Sistema Nacional Integrado de Cuidados que pretende modificar la actual división del trabajo, es que se plantea la necesidad de conocer cómo son los discursos de varones de la generación actual que son padres de niños/as menores de 6 años $y$ pertenecen a distintos niveles socioeconómicos.

\section{Tipos de paternidades}

Las paternidades y maternidades, en cierta medida podrían reproducir una dicotomía de género que ha sido cuestionada principalmente por los estudios de la filósofa Judith Butler. En la segunda edición de "Do Men Mother?" uno de los estudios pioneros sobre paternidades, Doucet (2018), reflexiona sobre la necesidad de reconocer que el cuidado ha sido una tarea principalmente realizada por mujeres y visibilizarlo a través del uso de la palabra "mothering", pero suma a esto estudios que permitan visibilizar la existencia o no de los cambios en las paternidades, considerando que las mismas no necesariamente 
pueden estar vinculadas a un sexo biológico. En este sentido, para el caso latinoamericano, Fuller (2000) identifica que la paternidad es una dimensión central en la vida de los varones y que tanto paternidades como masculinidades no responden a un único modelo. En las distintas etapas de la vida de los varones la masculinidad, la sexualidad, el trabajo y la pareja tienen significados y pesos distintos en la vida de los varones.

En trabajos europeos más recientes se encuentran ejercicios de tipologías de paternidades. Tobío et al. (2010: 104) propone la idea de que el padre proveedor y protector tradicional entra en conflicto con la idea de una paternidad afectiva, cercana $e$ igualitaria. El European Institute for Gender Equality define una "masculinidad cuidadora" como lo opuesto a la "masculinidad hegemónica", ya que se basa en el compromiso activo de los varones en el cuidado, en vez de su participación a través de la provisión económica. ${ }^{1}$

Bonino (2003:171) estudia las nuevas paternidades, sintetizando la existencia de al menos tres grandes modelos de paternidad en occidente: las paternidades decadentes, las paternidades indirectas y las paternidades cuidadoras (padres participativos/igualitarios y participativos/ayudantes).

Los modelos de paternidad propuestos por el autor señalan el tipo de participación que implica cada paternidad en relación al cuidado de los hijos/as, además de que cada uno de los modelos puede vincularse con determinados contextos históricos. Las paternidades en decadencia, en donde distingue al padre amo del padre "ley", marcan un patrón de ausencia del trabajo de cuidados, en donde los varones encarnan un modelo tradicional en el que detentan el poder familiar, imponen orden y son dueños del patrimonio material, genético y emocional.

Las paternidades indirectas se asocian al momento histórico en que aumenta la tasa de participación femenina, los divorcios y se diversifica la tipología de familias presentes en las sociedades.

1 Definición tomada de: http://eige.europa.eu/rdc/thesaurus/terms/1060. La traducción, no literal, es de las autoras. 
En este contexto los padres, convivan o no con los hijos, no se vinculan directamente con ellos. Es el momento en que las mujeres asumen la doble carga de trabajo sin instalar demandas y negociaciones en las parejas.

Finalmente, el modelo de padres cuidadores parece vincularse con el escenario socio-político actual en el cual las demandas por mayor equidad y participación de los varones en el cuidado son parte de los debates públicos. Algunos de estos se han transformado en la revisión de políticas de licencias y reglas del mercado laboral en relación a la compatibilización con las actividades familiares (reproductivas), así como los derechos de los varones a ejercer la patria potestad, etc.

Por otra parte, Marinho (2011: 143) en su análisis sobre las paternidades en el marco de la conyugalidad identifica la existencia de paternidades conjuntas, de apoyo, incentivadas, autónomas y electivas.

Su modelo de paternidad conjunta es similar a la definición de paternidades cuidadoras igualitarias de Bonino (2003: 176). En ella los valores, normas y finalidades del cuidado de los padres están orientados a compartir y participar: dedicar tiempo, estar disponibles para los hijos, repartir trabajo y tareas de cuidado con sus parejas. Es una paternidad construida en las dinámicas conyugales de parejas con representaciones sociales de lo que implica estar en pareja de tipo "compañerista": igualitario, dialogante, enfocado en la intensidad de la reciprocidad afectiva $e$ instrumental (Kellerhals et alii, 1982; Kellerhals; Widmer, 2005; Wall; Aboim; Cunha, 2010; Wall, 2005; Widmer; Kellerhals; Levy, 2003, en Marinho, 2011).

En las paternidades de apoyo (Marinho, 2011:164) el «apoyo» es la categoría discursiva que los encuestados utilizan para expresar esta inclusión multifacética del hombre en la producción de la vida cotidiana de los hijos y el compartir parental. En las prácticas de cuidado, participan activamente, pero de manera complementaria (apoyo a las madres).

Las paternidades incentivadas son otro modelo que pone de manifiesto que socialmente no basta con la provisión económica 
para sentirse padre, por lo que resulta importarte incorporarse de alguna manera en los planos más afectivos. En este tipo de paternidad dicha incorporación está mediada por las madres, ya que se refleja una mayor responsabilidad masculina, pero las mujeres siguen reduciendo sus jornadas laborales para cuidar, indicando que el modelo implica que es a ellas a quienes les corresponde el liderazgo en el cuidado y la organización doméstica, así como llevar a cabo la articulación entre el trabajo y la familia.

Las paternidades autónomas pueden ser igualitaria y apropiativa. La primera está anclada en valores y normas de una paternidad relacional y cercana, siendo la paternidad un ámbito de expresión de la autonomía del hombre en la esfera familiar, teniendo una relación individualizada con los hijos. La segunda se trata de la aparición de una rivalidad afectiva e identitaria del varón con su pareja. Se da un compartir parental basado en la apropiación valorativa y práctica (lugares, papeles, competencias y poderes en la familia, culturalmente asignadas a "lo femenino") por parte de los varones (Marinho, 2011: 211).

Las paternidades electivas se dan en torno a normas y valores de igualdad, autonomía e individualidad en relación a los hijos; pero el proyecto de ser un padre con una relación individualizada con los hijos/as se pone en competencia con ser un "hombre autónomo" que busca realizarse en proyectos profesionales y personales independientes de los familiares (Wall, Aboim; Marinho, 2007; 2010, en Marinho, 2011:156). En vez de unir estos ideales a las prácticas, como hacen los padres autónomos e igualitarios -que inscriben la autonomía, la igualdad y la individualidad en varias esferas sociales de realización de sí, tanto familiares como públicas- estos hombres vivencian la participación familiar en pequeñas dosis y privilegian su éxito profesional en detrimento del de sus socios, contribuyendo a la inversión desigual de los cónyuges en las esferas profesionales y parentales (Wall, 2010a, en Marinho, 2011:204). 


\section{Metodología}

Para dar cuenta del objetivo general que es comprender cómo significan las paternidades, los varones que son actualmente padres de niños/as de 6 años, el presente trabajo se basa fundamentalmente en el análisis de contenido de los discursos de varones de distintos niveles socioeconómicos.

A la vez, se plantea una descripción de los padres varones actuales para enmarcar las producciones discursivas en algunos datos generales en base a la Encuesta de Uso del Tiempo (2013).

El muestreo realizado fue teórico, no probabilístico ya que se seleccionaron unidades muestrales que responden a criterios categóricos (en este caso, nivel socioeconómico), acorde con los objetivos de investigación. Como en la mayoría de los estudios cualitativos, el presente busca generar validez interna y no externalizar los resultados en generalizaciones para grandes poblaciones (Padua, 1979:65).

La diferenciación de entrevistados por nivel socioeconómico obedeció a las diferencias en las estrategias de cuidado y sus representaciones sociales observadas en los antecedentes. Para dar cuenta de las mismas se definieron familias de nivel socioeconómico alto, medio y bajo, clasificándolas en función de las siguientes variables: para el caso de la clase alta se tomó como criterio la pertenencia a familias de altos ingresos y posesión de grandes extensiones de tierra en Uruguay, en los siguientes estratos la variable que primó fue el trabajo remunerado de la mujer y sus características (ocupación, profesión, dedicación, rama de actividad).

Las entrevistas a los padres varones actuales complementadas con las de abuelos (4) y bisabuelo (1), fueron parte de un estudio más grande que cuenta con 50 entrevistas a profundidad a tríadas de familias, que busca conocer el cuidado en tres generaciones. En dicha investigación se entrevistaron mujeres y varones a cargo del cuidado y por momentos se citarán mujeres como herramienta para la comprensión del fenómeno en la pareja. El artículo se centra en el análisis de los padres varones 
actuales por la alta tasa de no respuesta o no presencia de varones en las generaciones de bisabuelos (quienes habrían sido padres entre 1955 y 1965) y abuelos (1975-1990).

\section{De paternidades tradicionales a paternidades cuasi corresponsables: reproducción y cambios en las paternidades actuales}

En las entrevistas a profundidad se ha detectado una diversidad de discursos sobre el cuidado que evidencia una época de transición y transformación en los roles de género que no afecta a todos los varones y las mujeres por igual. Se han encontrado una serie de diferencias en la vivencia de la paternidad asociadas a los niveles socioeconómicos, en donde las personas más pobres reproducen conductas más tradicionales en la división sexual del trabajo y los roles de género en el cuidado, mientras que en los niveles económicos altos se encuentra una convivencia de elementos tradicionales con una leve participación más centrada en lo vincular con los niños, y en los sectores mediosprofesionales, más expuestos a un discurso de género, en donde se dan cambios más significativos en cuanto al involucramiento de los padres en el cuidado, tanto en el tiempo dedicado como en lo vincular.

A continuación, se muestra la distribución de varones en diferentes variables, según el tiempo que dedican al cuidado en la Encuesta de Uso del Tiempo (2013). ${ }^{2}$

\footnotetext{
2 Se sigue la metodología para establecer umbrales de pobreza de tiempo al tomar una vez y media la media o la mediana de tiempo dedicado al trabajo no remunerado (INMUJERES, 2012; Merino, 2010). No cuidadores: dedican 0 hora semana, cuidadores dedican hasta 21,9 horas semanales y de ese número en adelante, son cuidadores intensivos.
} 
Tabla 1. Varones no cuidadores, cuidadores o "cuidadores intensivos" con hijos de 6 años o menos por variables descriptivas. Total país, 2013

\begin{tabular}{|c|c|c|c|c|c|}
\hline & & $\begin{array}{c}\text { No } \\
\text { cuidadores }\end{array}$ & Cuidadores & $\begin{array}{l}\text { Cuidadores } \\
\text { "intensivos" }\end{array}$ & Total \\
\hline \multirow{2}{*}{ Edad } & Entre 18 y 29 & 23,6 & 22,6 & 15,0 & 23,4 \\
\hline & Entre 30 y 59 & 45,1 & 66,8 & 79,3 & 47,7 \\
\hline \multirow{4}{*}{ Nivel educativo } & Primaria & 26,1 & 23,7 & 17,8 & 25,7 \\
\hline & $\begin{array}{l}\text { Secundaria } \\
\text { básica Utu } \\
\text { equivalente }\end{array}$ & 34,4 & 38,4 & 14,4 & 34,5 \\
\hline & $\begin{array}{l}\text { Secundaria } \\
\text { superior Utu } \\
\text { equivalente }\end{array}$ & 21,4 & 24,6 & 42,3 & 22,0 \\
\hline & $\begin{array}{l}\text { Universitario o } \\
\text { más }\end{array}$ & 12,4 & 8,8 & 19,0 & 12,2 \\
\hline \multirow{5}{*}{$\begin{array}{l}\text { Categoría de } \\
\text { ocupación }\end{array}$} & $\begin{array}{l}\text { Asalariado } \\
\text { privado }\end{array}$ & 39,3 & 54,0 & 44,9 & 40,8 \\
\hline & $\begin{array}{l}\text { Asalariado } \\
\text { público }\end{array}$ & 8,6 & 9,8 & 20,1 & 8,8 \\
\hline & Patrón & 4,9 & 4,5 & 12,6 & 5,0 \\
\hline & $\begin{array}{l}\text { Cuenta propia } \\
\text { sin local ni } \\
\text { inversión }\end{array}$ & 1,3 & 2,3 & 4,9 & 1,5 \\
\hline & $\begin{array}{l}\text { Cuenta propia } \\
\text { con local o } \\
\text { inversión }\end{array}$ & 13,9 & 15,1 & 12,6 & 14,0 \\
\hline \multirow{3}{*}{$\begin{array}{l}\text { Modelo } \\
\text { Pareja }\end{array}$} & $\begin{array}{l}\text { Modelo } \\
\text { proveedor } \\
\text { tradicional }\end{array}$ & 26,3 & 37,0 & 12,2 & 27,3 \\
\hline & $\begin{array}{l}\text { Modelo } \\
\text { proveedor } \\
\text { modificado }\end{array}$ & 17,7 & 24,5 & 36,7 & 18,9 \\
\hline & $\begin{array}{l}\text { Modelo doble } \\
\text { carrera }\end{array}$ & 30,0 & 27,4 & 46,5 & 30,0 \\
\hline
\end{tabular}

Fuente: Elaboración propia en base a la Encuesta de Uso del Tiempo, INEINMUJERES-FCS. Uruguay, 2013. 
Cerca del $80 \%$ de los varones que se dedica intensivamente al cuidado infantil tiene entre 30 y 59 años. En la medida que los varones tienen menores niveles educativos se involucran menos en el cuidado de los niños/as (nótese que los varones universitarios están sobrerrepresentados en la categoría de "cuidadores intensivos", 19. La mayor proporción de cuidadores intensivos en empleos públicos podría asociarse a la estabilidad y el garantido goce de licencias que caracteriza al sector.

En cuanto a la provisión económica de los hogares, se observa que el modelo de proveedor tradicional (varón aporta la principal fuente de ingresos) es más preponderante entre los varones que no cuidan $(26 \%)$ y disminuye entre los cuidadores intensos $(12 \%)$, aunque aumenta en los cuidadores de niños/as que no son intensivos (37\%). Los varones que no cuidan a niños pequeños pertenecen menos a los modelos en donde hay una distribución del aporte de ingresos al hogar menos tradicional o de doble carrera, mientras que los cuidadores intensos forman parte de estos hogares. A continuación, se desarrolla de manera breve tres tipos de paternidades actuales como propuestas de categorías para pensar las paternidades actuales en Uruguay.

\section{Paternidades Tradicionales}

La presencia de la "Paternidad Tradicional" aparece principalmente en los niveles socioeconómicos más bajos, en donde los varones son "dueños y señores del hogar", mientras que las mujeres son un medio para la auto reproducción de los varones. No están muy interesados por el vínculo en la crianza, que es generalmente delegado a la madre, al menos mientras son niños pequeños (Clerget, 1992, en Bonino, 2003:173). Las mismas son similares a las identificadas por Bonino (2003) como paternidades decadentes.

Se trata de varones que tienen un discurso tradicional sobre la paternidad, un bajo involucramiento en el trabajo de cuidados ya sea el afectivo o el material, delegando en las mujeres (por 
considerarlas cuidadoras naturales) la gestión y ejecución del mismo.

Este tipo de paternidad, que responde a una división sexual del trabajo, en generaciones pasadas no era un aspecto a cuestionar ya que se asumía el deber ser de las mujeres en torno al cuidado sin mayores cuestionamientos (Batthyány; Perrotta; Scavino, 2017:78).

Y no, no, son más dramáticas, son más cuidadoras, son más protectoras, es diferente, nosotros por ejemplo ta lo vemos llorar lo miramos y vemos si está llorando maña o está llorando por hambre. Ella ya es diferente si llora ah mira como esta y ya es diferente el cuidado, no? (Decís que se conecta mejor con el niño la mujer).Claro, se conecta en el sentido que le da más maña, lo cuida más es más protectora. Los varones, lo cuidamos a lo justo y necesario como quien dice (Padre, NSE Bajo).

Sin embargo, cuando se les pregunta si están conformes con la distribución de las tareas de cuidado afirman que sí, pero advierten (casi como comete una falta) que ni quieren saber lo que opinan sus compañeras: "Sí obvio, yo estoy con conforme, te dejo con mi señora después no quiero ni enterarme tampoco, jeje". (Padre, NSE Bajo).

Este discurso es acompañado por la naturalización que las propias madres tienen sobre ellas mismas como principales cuidadoras:

No, obligada no, pero... sí, soy la madre, o sea, es como que... Sí. Ya te digo, compartimos pila de responsabilidades con (Pareja), ¿no? pero sí, en el día, cuando estoy yo, la responsable soy yo, o sea. (...) como que si... la responsable soy yo. Y sí me siento como... (pausa) sí, obligada a si a cuidarlo y a todo, ¿no? (Madre, NSE Bajo).

Los mismos coinciden con los datos de la Encuesta Nacional de Adolescencia y Juventud (2013) que muestran que las mujeres 
del quintil más bajo de ingresos son las que están de acuerdo en mayor proporción con la sentencia "criar a los hijos es tarea primordial de las mujeres". Esta introyección de los mandatos de género clásicos contribuye para comprender que, en un contexto donde las desigualdades de género son un tema politizado y asumido como una responsabilidad del Estado, la corresponsabilidad en el cuidado esté aún lejos de ser real.

Si bien en la modernidad, imponer orden y conductas en los niños era parte de lo que los varones y las mujeres tenían que hacer en la crianza (padre patrón-educador, según Bonino (2003), los varones tomaban el lugar del "extraño" del "outsider" a quien había que respetar. En las paternidades actuales que hemos llamado tradicionales, aparecen rastros de esta autoridad en las amenazas a los niños/as como formas de crianza: "cuando venga tu padre vas a ver". Esta autoridad deviene de la ausencia en la vida cotidiana más presente en hogares de niveles socioeconómicos bajos donde la división sexual del trabajo es, frecuentemente, más clásica (varón proveedor, mujer ama de casa).

No, no él en realidad como que no opina mucho, o sea él me da siempre el sí a lo que yo elijo, yo decido algo y él siempre me da el sí, hay que hacer algo y siempre me pregunta a mi primero, siempre es así la que decide más bien soy yo entonces ta él está. Ahora con esto también me dijo que está muy de acuerdo, él ya me ha dicho no vayas más quédate con el gordo, digo él está de acuerdo, no tiene problema (Madre, NSE Bajo).

En estos contextos, la participación de los varones en las tareas domésticas y de cuidados es puntual, principalmente durante los fines de semana y su ausencia en estos trabajos es justificada en los discursos a partir del hecho de que trabajan remuneradamente (muchas veces no tienen trabajos estables.

Y no, en realidad lo cuida más ella que yo porque yo más bien vengo de noche (de trabajar) y de noche cuando estoy 
desvelado, estoy de noche acá con ella mirando tele y lo que sea y ta después yo me levanto tarde y ella por lo general lo cuida todo el tiempo (...) Lo asume más ella que yo, eso. (Varón, NSE Bajo).

Si bien los varones padres ausentes son "descastados" de un rol de padre con funciones o sentidos claros, los mismos decodifican y justifican su ausencia en la crianza: "Ella se encarga de lo doméstico como quien dice y aparte ella esta acá todo el día con él y entonces lo conoce más que yo" (Varón, NSE Bajo). Buena parte de las mujeres de hogares más pobres se mantienen por fuera del mercado laboral en los primeros años de vida de los/as niños/as. En buena medida por el costo de oportunidad, aspecto que es reproducido por las políticas actuales que basan su diseño en el supuesto de que hay alguien que se hace cargo del cuidado de los hijos/as en el hogar, en horarios laborales. Esto se acompaña de la ausencia de habilitaciones materiales y simbólicas para que los varones dispongan de tiempo para dedicarse al cuidado de los hijos, y para que las mujeres cuenten con herramientas que les permitan elegir si cuidar o no y manejar con mayor libertad los tiempos. En medida que esto siga ocurriendo persistirán barreras culturales y de oportunidades para ejercer otro tipo de masculinidad diferente a la hegemónica (Connell, 1995, en Comas, 2016:11).

En resumen, hoy en día existen principalmente en los niveles socioeconómicos bajos paternidades de tipo tradicionales que heredan de otras épocas la presencia de padres "amos" (uno de los tipos de paternidad en decadencia presentada por Bonino, 2003), que son quienes imponen la ley en el hogar, se plantea un tipo de división sexual del trabajo clásica que aparece justificada por la asunción de la provisión económica como aspecto ineludible por parte de los varones en el afán de cumplir con su rol de "varón" socialmente asignado. Este varón proveedor que no puede dedicarse al cuidado y las tareas domésticas, sigue siendo un rasgo de la masculinidad hegemónica actual, que aparece de 
manera más difusa en los varones de la generación de padres actuales.

Sumado a esto, el contexto de instituciones y políticas, si bien ha avanzado, lo ha hecho de manera segmentada ya que los padres actuales de niveles bajos cuentan con menores incentivos para considerar su participación en los cuidados que devenga de las políticas de trabajo-familia, particularmente por su sobrerrepresentación en los empleos informales y precarios "Y cuando nació: ¿pudiste tomarte licencia paternal o por paternidad? Ah, no me acuerdo (...) cuatro días, no estaba en este laburo estaba en la construcción (...) Sin pago nomás" (Varón 1, NSE Bajo).

Así, las paternidades tradicionales a las que no se les puede demandar tiempo y dedicación para los cuidados de los hijos, no solo se anclan en los sujetos, sino que las instituciones también impiden el desarrollo de las mismas. La masculinidad hegemónica coincide con una naturalización de las mujeres como cuidadoras y un fuerte familismo en los cuidados, generando que los varones sean "privilegiados irresponsables" (Tronto, 1999). Esto último quiere decir que han estado eximidos del cuidado, no considerándolo su responsabilidad, a la vez que estos padres gozan de mayores niveles de autonomía en casi todas las áreas de la vida social, en gran medida, por no asumir los costos del cuidado.

\section{Paternidades de involucramiento leve}

Un segundo modelo es el de las paternidades de "involucramiento leve". El mismo es esencialmente un modelo de paternidad tradicional al que se suma un involucramiento en el cuidado desde el juego, los paseos y centrado principalmente en lo afectivo, una valorización sobre estar presentes en lo educativo acompañado de una reflexividad sobre la paternidad (presente y en retrospectiva). Este tipo de paternidades aparece en los padres actuales de niveles altos y algunos de niveles económicos medios altos. 
La figura central en este tipo de paternidad es "la ayuda", por lo que presentan características similares al modelo de paternidades de apoyo esbozado por Marinho (2011) que, a su vez, son similares a las propuestas por Bonino (2003:4) presentadas como cuidadoras (dice "las paternidades cuidadoras son mayoritariamente participativos-ayudantes $y$ no tanto igualitarios) una subdivisión de las paternidades cuidadoras, en donde los varones cuidan, pero desde un lugar marginal.

A diferencia de las paternidades tradicionales, las paternidades de involucramiento leve captan las necesidades de cuidado de los hijos, que delegan a las mujeres cuando son materiales, pero deciden prestar atención a los elementos afectivos del cuidado. Es importante resaltar que, en el NSE Altos, la resolución del trabajo material de cuidados casi siempre está en manos de una mujer más pobre contratada para la tarea.

En las paternidades de involucramiento leve, aparecen el dialogo entre padre e hijos como elemento central en el discurso sobre el cuidado: "El diálogo con los chicos es fundamental" (Padre 1, NSE Alto). Incluso, los padres actuales se comparan con sus propios padres a quienes perciben como más autoritario y ausentes que ellos en la crianza.

Sí, creo que hay mucho más diálogo padre-hijo y mucho menos miedo a contarse las cosas que antes. (...) Pero ahora lo veo, vamos a comer los domingos y él en un momento pretendió ser el abuelo omnipotente y lo bajaron de un plumazo. No va más eso" (Padre 1, NSE Alto).

A su vez, expresan la voluntad de lograr equidad en la pareja brindando un cuidado de calidad: "Básicamente, eso: dividirnos el tiempo entre los dos, tratar de pasar la mayor cantidad de tiempo y la mejor, o sea, quality" (Padre, NSE Alto).

Sin embargo, en el mismo discurso se presenta que si bien se capta la necesidad de cuidado (hacer las cadenas para ir al colegio) y se responsabiliza un tiempo por el mismo, luego se vuelve a delegar a las mujeres, mostrando que el discurso de 
"equidad" que presentaba más arriba no coincide con sus prácticas, tal como muestran los antecedentes cuantitativos (Hakim, 2005:80; CIS, 2004b:1, en Tobío, 2010:105; Batthyány; Ferrari; Scavino 2015:161).

Esto también sucede con los varones de niveles socioeconómicos bajos.

No soy un tipo machista, para nada, no sé qué te dijo ella (risas). Nos llevamos creo que muy bien, pero sí, sí, claramente hay cosas de las que se ocupa ella, así como hay cosas de las que me ocupo yo. A ver, cuando hay reuniones de padres en el colegio, trato de ir a todas, pero sí, llevarlos al médico, al dentista, yo qué sé, cosas que son a las 3, 4, 5 de la tarde y yo estoy acá... las cadenas, el año pasado las hice yo todas, todo el año, y este año dijimos bueno, pará, necesito un poco de tiempo en la mañana para al menos salir a andar en bici o correr un poco o moverme algo, porque hay que cuidarse. Entonces bueno, este año (nombre pareja) está haciendo las cadenas, que le agradezco pila, porque la verdad es que son... (Padre 1, NSE Alto).

En el discurso se observa claramente como ante la responsabilidad del cuidado, los varones no se hacen cargo, delegando en las mujeres el trabajo y su organización, quienes a su vez cuentan con los privilegios de poder tercerizar una gran parte del cuidado (el rutinario y "sucio") contratando a otras mujeres para su realización. En este sentido, la mayor carga de cuidado en estos sectores económicos está siendo asumida por mujeres pobres que son contratadas como niñeras o cuidadoras por las mujeres de estas parejas.

La pareja se presenta como un ámbito de negociación y acuerdos mutuos en lo que respecta al cuidado y las tareas de cada uno ("somos un equipo eso es nuestra teoría acá somos un equipo vamos para el mismo lado" (Madre 1, NSE Alto)). Así, a diferencia de las generaciones anteriores, se sostiene una clásica división sexual del trabajo, pero está mediada por una ideología 
sobre la maternidad y por acuerdos en la pareja. Las mujeres de la pareja demandan que lleguen más temprano a la casa, o a veces, resuelven conflictos asumiendo las tareas ellas: "porque soy menos calentona... entonces ya no se lo exijo, en un momento se lo exigí (...) pero bueno ta, había que estar" (Madre 1, NSE Alto).

Sin embargo, mientras que para ellas hay conflictos y demandas en cuanto a quién cuida y quien trabaja, para ellos las divisiones se han dado naturalmente: "Se fue dando naturalmente. Es más, yo, por lo menos, nunca nos cuestionamos nada" (Padre, NSE Alto).

En las paternidades de involucramiento leve, el argumento del trabajo remunerado también es una explicación de la ausencia en los cuidados, aunque el mandato del trabajo se relativiza:

si viviera en una utopía, sería no tener que trabajar, hacer así y que me cayera la plata, y que pague el colegio, MP, esto, lo otro y pasar todo el tiempo bien, felices, familia Ingalls y chau, pero no es así (...) Me gustaría estar un poquito más presente, porque cuando llego, llego $7 \mathrm{y}$ media, 8, y estamos una horita, y estoy con el enano, los otros dos grandes ya se meten en su cuarto, computadora... pero, bueno, no me puedo quejar para nada; son un agradecido de la familia que tengo (Padre 1 , NSE Alto).

Aunque asumen como natural el proceso por el cual las mujeres de la pareja quedan por fuera del mercado laboral:

Por suerte, nunca tuvo que salir ella a buscar un trabajo y a emplearse en un lugar. (...) Siempre voluntad de ella, decisión de ella y siempre hizo lo que quiso en ese aspecto. (¿Y esa decisión de no salir a trabajar fue algo como conflictivo o...?) No, no fue ni hablado, fue natural. Para nada (Padre, NSE Alto).

En resumen, los padres de niveles socioeconómicos altos y medios-altos, sostienen una masculinidad tradicional y son los 
principales proveedores de ingresos, pero han cambiado su visión sobre lo que implica ser padre. Hay una preocupación por lo afectivo que aparece como válida y una potenciación en el discurso de la "calidad" en los cuidados, más que cantidad (aspecto que sigue relegado a las mujeres). En este tipo de paternidad de involucramiento leve, los varones se involucran en el juego, los paseos y las tareas de llevar y traer, siendo auténticas paternidades de apoyo (Marinho, 2011). Pero además reflexionan sobre cómo ser padres, piensan por ejemplo en charlar con sus hijos antes de reprenderlos. Lo hacen por oposición a la imagen de ley y de orden (de castigador) que tienen de sus propios padres, buscando desmarcarse de ese tipo de ejercicio de la paternidad. Es decir que tienen reflexión en base al ejercicio de la paternidad y consideran que es importante estar cerca de los hijos para dialogar con ellos y educarlos. Además, se diferencian del modelo de masculinidad hegemónica de sus propios padres ("él en un momento pretendió ser el abuelo omnipotente y lo bajaron de un plumazo. Eso no va más") y tienen un discurso equitativo de género. En cuanto a las tareas que realizan, como se ha mencionado, es clasificada como una paternidad incuestionable modificada debido a que se interesan por los hijos y los elementos afectivos del cuidado, involucrándose cuando quieren en las tareas que quieren, pero se delega en las madres toda la organización, el pienso y el hacerse cargo del cuidado.

\section{Paternidades cuidadoras (con tendencia a la corresponsabilidad)}

Finalmente, existe un grupo de padres cuidadores, que si bien no son corresponsables igualitarios en el sentido de Bonino (2003:176), tampoco son ayudantes: son padres que se involucran activamente en el cuidado, que dedican tiempo al mismo y asumen el desafío de hacerse cargo de las tareas de manera "autónoma". Sin embargo, admiten que en algunos aspectos las madres son quienes tienen la estrategia completa de cuidados en su mente (cuidado intangible). 
Este tipo de paternidades se encuentra fundamentalmente en los niveles económicos medios (medios-medios y medios-altos) de los padres actuales. Se diferencian de modelos anteriores, principalmente en la asunción de la realización de tareas de cuidado de "mano propia" por parte de los varones, entendidas como algo importante en el ejercicio de su paternidad. Estos padres no solo reflexionan sobre lo que implica el vínculo de cuidados y la realización del mismo, sino que además ponen de manifiesto los obstáculos que las políticas de cuidado (licencias, servicios) imponen al ejercicio de su paternidad, así como piensan al reparto equitativo como importante para el desarrollo de ambos miembros de la pareja en otras esferas de su vida (trabajo, estudios, ocio).

Los padres cuidadores son los "nuevos padres", categorizados así por Abril et alii (2015). Tobío afirma que los padres implicados en el cuidado de manera igualitaria representan un futuro minoritario, pero que van en una línea coherente con los cambios familiares descrito al principio por los que han transitado las sociedades occidentales (Tobío, 2010:108).

Uno de los elementos que hace que se diferencien de las paternidades tradicionales y de involucramiento leve es la desnaturalización del cuidado asociado a lo femenino.

Y lo que toca hacer, lo hacen con quien estén, no hay nada que sea exclusivo de decir no mira, esto lo haces con mama o cuando venga mamá te bañas, o te hace la comida o lo que sea, no, no, con eso no, no (Padre, NSE Medio).

Esto también es considerado por las mujeres: "No. No, no. No, responsabilidad ninguna porque me parece que hoy en día la mujer tiene que hacer lo mismo que el hombre" (Madre, NSE Medio).

En general, se intenta llegar a acuerdos en la distribución de manera que los proyectos profesionales, laborales y educativos de ambos no se vean truncados por las tareas de cuidado, sin embargo, esto implica una ingeniería compleja, particularmente en 
los casos con los que no se cuenta con una mercantilización de las tareas domésticas o el trabajo de cuidados.

Nos costó, nos costó discusiones, idas y venidas y ahora está como un poco más armónico. (...) No sé, para darte un ejemplo ahora saca la ropa y la dobla. Antes sacaba la ropa y la dejaba arriba de la cama, ¿qué pensás, que se dobla y se guarda sola? Hacés toda la tarea hacela entera, porque yo no te saco tu ropa y la dejo tirada arriba de la cama para que se doble. La doblo, la guardo. Como que en eso nos fuimos entendiendo. Y bueno, ta. Fue como un proceso (Madre, NSE Medio).

La idea de proceso y aprendizaje están vinculadas a las demandas de las mujeres hacia los varones para el establecimiento de acuerdos en la convivencia. Cuando la distribución no es tan equitativa, también hay una reflexión de las mujeres sobre los límites que anteponen al involucramiento de los varones en el cuidado.

Yo creo que es más un tema mío y de que yo no lo dejo ser, por querer hacerlo más rápido, a que él no quiera hacerlo. Cuando lo ha tenido que hacer lo hace y lo hace bien. Creo que es eso (Madre, NSE Medio).

Las mujeres no solo se reconocen a ellas mismas como reproductoras de un orden de género que ponen en cuestión, sino que también se permiten demandar corresponsabilidad en los cuidados. Aun cuando el tenor es de intento de repartir las tareas de manera equitativa las mujeres relatan planteos de demandas.

Sí... sí, sí, eso mil veces se ha planteado, yo pila de veces le he planteado cosas que yo sentía, que estaba como más sobrecargada, y con total apertura, ¿no? siempre me escuchó y trató de cambiarlo, de modificar (Madre, NSE Medio). 
También, existe la posibilidad de ser reflexivos y buscar modelos alternativos para poder cuidar, una de las estrategias que se propone es reducir la carga horaria de los empleos de ambas parejas para destinar más tiempo al trabajo no remunerado procurando distribuir equitativamente las tareas entre ambos sexos.

ella muchas veces me ha dicho que por qué no dejar los trabajos y trabajar por nuestra cuenta en casa, pero... como que a veces lo manejamos como un ideal, pero también lo vemos como medio lejano (...). Sí, yo a veces siento la necesidad de estar más en mi casa (Padre, NSE Medio).

Otra dimensión importante en las paternidades con tendencia a la corresponsabilidad es el valor que los varones otorgan a estar presentes en el cuidado y ser afectivos.

Bien. ¿Y qué tareas realizas en la cotidiana? Escucharlos, estarles corriéndolos atrás para que levanten las cosas, la leche, el desayuno de mañana, insistirles justamente para que se laven los dientes, para que mantengan un poco el orden... O sea estarles un poco atrás y hacer el almuerzo. $\mathrm{O}$ sea, mientras que terminan de desayunar y se van vistiendo y de más, y algún juego corto que surge... (Padre, NSE Medio).

En los casos en que las mujeres trabajan más tiempo, los varones se encargan del cuidado, pero esto probablemente se vincule también a los ingresos que provee cada uno. A veces, la participación es mutua y esto se asocia a los horarios de estudio y de trabajo de la madre.

si él estuvo todo el día con el padre en mi casa y yo llego de trabajar, bueno el padre se pone a cocinar y yo me dedico a jugar con él y a bañarlo y a estar con él y a hacerlo dormir (...) y luego establece que cuando están los dos juntos "Compartimos todo. Él lo baña, le da de comer, yo lo 
baño, le doy de comer, eh... Jugamos los dos (Madre, NSE Medio).

Sin embargo, estas nuevas paternidades suceden en contextos en los que las instituciones, particularmente las del mercado laboral, reproducen las lógicas que postergan una división sexual del trabajo clásica y un modelo de varón "dedicado $100 \%$ al trabajo remunerado". Dentro de las diferentes reglas que se aplican para uno y para otros respecto a su inserción en el mercado laboral, se encuentra que mientras que el trabajo del varón es "intocable", la madre tiene que tener disponibilidad para siempre poder llevar a cabo la tarea acordada, o como dice una entrevistada, "no me puede pasar nada", porque si bien las urgencias por trabajo son admitidas para los varones, para ellas no.

Eh... sé que yo no puedo tener imprevistos como puede tener él, ¿̇me entendés? (...) Porque donde me pase ya no sé quién los levanta. ¿Entendés? En ese sentido sí creo que... en mi caso "pareja" está muchísimo más descansado. Porque él sí puede tener un imprevisto. Llegar a las siete, "tuve una reunión, salí más tarde" (Madre, NSE Medio).

Vinculado a esto, es destacable que en los discursos de los varones del sector medio también plantean una extensión de las licencias por maternidad o paternidad de manera de que alguno de los dos pueda estar el mayor tiempo posible en los primeros meses de vida del bebé. Desde un discurso transformador de género se plantea que dicha extensión debe colocar en un mejor lugar al papá ya que lo que se espera como sociedad es la igualdad de género y la corresponsabilidad.

Que sea el padre o la madre pero que alguien de los dos pudiera estar más en su casa con el niño, sobre todo en la temprana edad. Acá en "EMPRESA" hay un beneficio extra que son 8 días. Pero digo, más allá de que está muy lejos de lo que es la legal, es más beneficioso, pero es muy 
poco...primero porque creo que así como estamos tratando de ser una sociedad igualitaria en género, es necesario que el padre tenga un derecho también, que creo que lo hace más lejano por eso, ese vínculo lo hace más lejano, no ayuda a eso...y además porque la madre en ese sentido es...en los primeros 3 meses de vida, la más afectada físicamente y emocionalmente, entonces tampoco sirve para ayudar en eso. O sea que prácticamente no sirve para nada la licencia por paternidad, y bueno...fue lo que tuvimos, y después la legal de la mamá (Padre, NSE Medio).

Por otra parte, se plantean demandas de medidas correspondientes a reducir la jornada laboral a 6 horas durante los primeros años de vida del niño, así como que el Estado incremente la oferta de escuelas de tiempo completo para evitar el pago de escuelas privadas que permitan la conciliación con la jornada laboral.

Creo que la educación pública tiene que adaptarse de una vez por todas a toda esta forma de vida, porque esto no va a cambiar, la forma de vida que tenemos hoy, y está bien que no cambien algunos aspectos, pero sí tiene que adaptarse la enseñanza pública, la escuela pública, porque creo que lo demás no...no sé si está tan lejos de la realidad como es la escuela, sobre todo en la escuela es terriblemente ineficiente, porque termina siendo algo que no colabora. No digo que en el $100 \%$ pero en las clases medias seguro que no, por los...el armado familiar que tenemos hoy...y bueno, tienen que hacer extensiones horarias que sirvan y que sean reales a...también hay cosas que a los privados que se les pueden exigir, y eso tiene que salir del Estado también, como por ej., leyes que apoyen la maternidad o la paternidad y que las empresas las obliguen, empresas sobre todo que pueden hacerlo (Padre, NSE Medio).

En resumen, si bien no hay padres absolutamente corresponsables, se encuentran padres de niveles económicos 
medios que se involucran más allá de la figura de ayudantes. Son padres que o bien tienen trabajos que les permiten estar más presentes en la esfera doméstica por su flexibilidad o que tienen horarios fijos pero que reconocen y reclaman por las limitaciones que el mercado laboral y la ausencia de políticas imponen al ejercicio de su paternidad.

Hay un involucramiento directo en las tareas de cuidado más allá del juego y del acompañamiento, lo cual hace que estos padres comprendan las implicancias y costos del cuidado. Algunos se han planteado reducir sus jornadas laborales para poder cuidar más, lo cual va en contra del tipo de paternidad tradicional en donde los padres son los proveedores económicos principales. Si bien en la mayoría de los casos los padres con tendencia a la corresponsabilidad, tercerizan parte del tiempo en la contratación de centros privados de cuidado infantil, es durante el tiempo que padre o madre deben hacerse cargo del cuidado, en donde es notorio su mayor involucramiento en relación a los otros dos tipos de paternidades identificados.

La presencia de estos padres hace que los mismos no sean una fuente de amenaza que los coloque como la autoridad (que impone la ley y el orden) hacia los niños/as. Sino que son padres más cercanos e involucrados activamente en los procesos de crianza.

Estas paternidades se dan en el marco de parejas que son negociadoras que experimentan conflictos y tensiones en relación a las responsabilidades de ambos y a los acuerdos de convivencia. Las parejas son espacio de aprendizaje en la búsqueda de una distribución más equitativa del trabajo de cuidados, presentándose una reflexividad sobre las prácticas de cuidado.

\section{Reflexiones finales}

En el presente artículo se ha llevado a cabo una primera tipología de paternidades en el Uruguay actual, a partir de la reflexión sobre el papel de los varones en el cuidado que emerge principalmente del discurso de varones padres de niños de 6 años 
o menores. A partir de la reflexión teórica y el análisis de contenido de los discursos se han generado tres grandes tipologías de paternidades: las paternidades tradicionales, las de involucramiento leve y las cuidadoras (con tendencia a la corresponsabilidad). En la tabla 6 se resumen las características de las mismas en distintas dimensiones.

La detección de estas tipologías de paternidad muestra un panorama muy heterogéneo en relación a la posición de los varones respecto al cuidado en la sociedad uruguaya actual, pero particularmente llama la atención la fuerte asociación de las tipologías con los niveles socioeconómicos.

Las resistencias del modelo tradicional en los niveles más bajos alerta sobre la necesidad de generar políticas que trabajen sobre la subjetivación de género tanto de varones como de mujeres, para lograr repartos más equitativos de los trabajos de cuidado. A su vez, se requieren mayores políticas de inclusión laboral que contribuyan y garanticen el ejercicio de la paternidad en los varones. Esto implica pensar en que la mayoría de los padres más pobres tienen vínculos informales con el mercado laboral, por lo tanto, la política no puede ser erigida solo desde los trabajos formales, sino que se necesitaría pensar en mecanismos alternativos para el fomento de la corresponsabilidad.

En segundo lugar, los padres de niveles socioeconómicos altos si bien tienen una estructura tradicional en el reparto de tareas, repudian las paternidades ausentes y distantes, y se han involucrado en el juego, los paseos y las actividades de traslados, aunque no se responsabilizan por el cuidado. Las mujeres son quienes sostienen el funcionamiento de la esfera doméstica toda, pero esto no es naturalizado por los varones, se entiende como un reparto que se da, principalmente vinculado a las diferencias en la provisión económica de ellos y ellas. Un aspecto importante de las paternidades de involucramiento leve es que si bien no son equitativas, siguiendo la hipótesis de que las clases altas son modelos de "ser" para otras clases, al menos estos padres consideran que estar por fuera del cuidado, no dialogar con los hijos o no verlos frecuentemente, es algo negativo. Si bien no es 
un cambio suficiente en términos de la equidad de género, al menos es un cambio en relación al modelo tradicional que genera costos más duros tanto para varones como para mujeres.

Finalmente, en los niveles socioeconómicos medios es donde se presentan las paternidades cuidadoras. Como se ha dicho, no son corresponsables igualitarias, puesto que las mujeres son quienes realizan más tareas de cuidado o quienes se encargan del cuidado intangible. Pero este es el modelo en donde los padres se hacen cargo del cuidado de manera activa y autónoma y se involucran en tareas cotidianas y rutinarias, más allá del juego, los paseos o los traslados que son actividades más placenteras y de las cuales se puede prescindir.

Estas paternidades cuidadoras se desarrollan por parte de varones reflexivos sobre el cuidado, de niveles educativos altos y que visualizan y relatan las limitantes de la política y del mercado laboral para el ejercicio pleno de sus paternidades (paternidades reclamantes, según Bonino (2003).

Son paternidades que se desarrollan en parejas que son construidas cotidianamente, y que se presentan como ámbitos de negociación y de reflexión. Se caracterizan por ser parejas en donde ambos son importantes en la provisión económica del hogar y el proyecto laboral o profesional de ambos es importante (de las mujeres y de los varones).

Del diverso panorama de paternidades en la actualidad evidenciadas en el presente trabajo se desprenden muchas preguntas en cuanto a las dinámicas de las parejas, los desafíos de la política para la comunidad, así como sobre la necesidad de promover la investigación sobre las nuevas paternidades en relación al cuidado en Uruguay.

\section{Referencias bibliográficas}

ABRIL, Paco et alii. Ideales igualitarios y planes tradicionales: análisis de parejas primerizas en España. Revista Española de Investigaciones Sociológicas, 150, 2015, pp.3-22. 
AgUAYO, F.; BARKER, G. (coord.). Masculinidades y políticas de equidad de género: Reflexiones a partir de la Encuesta IMAGES y una revisión de políticas en Brasil, Chile y México. Rio de Janeiro, Promundo, 2011.

Aguayo, F.; Barker, G.; Kimelman, E. Paternidad y Cuidado en América Latina: Ausencias, Presencias y Transformaciones. Masculinities and Social Change, 5(2), 2016, pp.98-106. [doi:10.17583/MCS.2016.2140].

BATTHYÁNY, Karina; FERRARI, Fernanda; SCAVINO, Sol. Juventud, representaciones sobre el trabajo no remunerado y brechas en el uso del tiempo. Dichos y hechos. En: BOADO, Marcelo (coord.). El Uruguay desde la Sociología 13. Montevideo, Doble Clic, 2015, pp.147-166.

BARBETA-ViÑAS, Marc; CANO, Tomás. ¿Hacia un nuevo modelo de paternidad? Discursos sobre el proceso de implicación paterna en la España urbana. Revista Española de Investigaciones Sociológicas, 159, 2017, pp.13-30.

Bátthyany, Karina; Genta, Natalia; Perrotta, Valentina. Avanzando hacia la corresponsabilidad en los cuidados: análisis de las licencias parentales en el Uruguay. Santiago de Chile, CEPAL, 2015.

BATTHYÁNY, Karina; GENTA, Natalia; SCAVINO, Sol. Análisis de género de las estrategias de cuidado infantil en Uruguay. En: BOADO, Marcelo (coord.) El Uruguay desde la Sociología 14. Montevideo, Doble Clic, 2016, pp.113-132.

BÁtThyanY, Karina; PerRotTA, Valentina; SCAVINO, Sol. El trabajo remunerado de las mujeres madres a lo largo de tres generaciones ¿un camino hacia la igualdad de género? En: PUCCI, Francisco (coord.) El Uruguay desde la sociología 15. Montevideo, Doble Clic, 2017, pp.67-80.

BÁtThyany, Karina; GentA, Natalia; PerrottA, Valentina. Uso de Licencias Parentales y Roles de Género en el Cuidado. Montevideo, SNIC, 2018.

BonINO, Luis. Las nuevas paternidades. Cuadernos de Trabajo Social 16, Madrid, 2003, pp.171-182. 
COMAS D'ARGEMIR, Dolors. Hombres cuidadores: Barreras de género y modelos emergentes. Psicoperspectivas, 15(3), 2016, pp.10-22.

DOUCET, Andrea. Do Men Mother? Fathering, Care, and Parental Responsibilities. 2nd Edition. Toronto, University of Toronto Press, 2018.

Fuller, N. Introducción. En: Fuller, N. (ed.). Paternidades en América Latina. Lima, Fondo Editorial de la Pontificia Universidad Católica del Perú, 2000, pp.11-32.

MARINHO, Sofía. Paternidades de hoje. Significados, práticas e negociações da parentalidade na conjugalidade e na residência alternada. Tesis (Doctorado en Ciencias Sociales), Lisboa, Universidad de Lisboa, 2011.

PADUA, Jorge. Técnicas de Investigación Aplicadas a las Ciencias Sociales. México, Fondo de Cultura Económica, 1979.

SCAVINO, Sol. Cuidados y subjetivación de género. Un análisis de discurso de las mujeres que constituyen hogares monoparentales con hijos pequeños. Psicología, Conocimiento y Sociedad, 7(2), 2017, pp.141-168.

SNIC. Informe anual 2017. Montevideo, SNIC, 2017.

TOBío, Constanza et alii. El cuidado de las personas. Un reto para el siglo XXI. Barcelona, Fundación "La Caixa", 2010. 